\title{
Spacing patterns on tongue surface-gustatory papilla
}

\author{
HAN-SUNG JUNG* ${ }^{*}$ KEIICHI AKITA ${ }^{1}$ and JAE-YOUNG KIM \\ Division of Anatomy and Developmental Biology, Department of Oral Biology, Research Center for Orofacial Hard \\ Tissue Regeneration, Oral Science Research Center, College of Dentistry, Brain Korea 21 Project for Medical \\ Science, Yonsei Center of Biotechnology, Yonsei University, Korea. ${ }^{1}$ Unit of Biostructural Science, Graduate \\ School, Tokyo Medical and Dental University, Bunkyo-ku, Tokyo, Japan
}

\begin{abstract}
The dorsal surface of the mammalian tongue is covered with four kinds of papillae, fungiform, circumvallate, foliate and filiform papillae. With the exception of the filiform papillae, these types of papillae contain taste buds and are known as the gustatory papillae. The gustatory papillae are distributed over the tongue surface in a distinct spatial pattern. The circumvallate and foliate papillae are positioned in the central and lateral regions respectively and the fungiform papillae are distributed on the anterior part of the tongue in a stereotyped array. The patterned distribution and developmental processes of the fungiform papillae indicate some similarity between the fungiform papillae and the other epithelial appendages, including the teeth, feathers and hair. This is because 1) prior to the morphological changes, the signaling molecules are expressed in the fungiform papillae forming area with a stereotyped pattern; 2) the morphogenesis of the fungiform papillae showed specific structures in early development, such as epithelial thickening and mesenchymal condensation and 3) the fungiform papillae develop through reciprocal interactions between the epithelium and mesenchymal tissue. These results led us to examine whether or not the early organogenesis of the fungiform papillae is a good model system for understanding both the spacing pattern and the epithelial-mesenchymal interaction during embryogenesis.
\end{abstract}

KEY WORDS: embryonic development, epithelial appendage, fungiform papillae, gustatory papillae

\section{Introduction}

During mouse embryogenesis, the terminal sulcus divides the developing tongue into an oral part and a pharyngeal part. Four different types of tongue papillae can be found on the dorsal surface: fungiform, circumvallate, foliate and filiform papillae. These papillae are distributed in a specific pattern (Iwasaki et al., 1999). The fungiform, foliate and circumvallate papillae are known as the gustatory papillae, which contain taste buds and work as sensory organs (Mbiene et al., 1997). The gustatory papillae are comprised of an epithelial covering over a broad core of connective tissue. Taste buds, which are discrete collections of approximately 40 to 60 cells within the papilla epithelium, are an oval sensory end organ that is involved in the perception of chemical stimuli and in taste transduction (Barlow and Northcutt, 1998; Oakley, 1998; Barlow, 1999).

The fungiform papillae are located on the oral part of the tongue with a specific patterned array. One circumvallate papilla is located in the middle part of the terminal sulcus in rodents. In humans, about 7 to 9 circumvallate papillae are located along the terminal sulcus (Fig. 1). Foliate papillae are found on the sides of the posterior one-third of the human tongue and are well developed at birth, but revert to a rudimentary structure in adults. The filiform papillae, which contain no taste buds, cover the entire anterior part of the dorsal surface of the tongue and consist of cone-shaped structures, each with a core of connective tissue that is covered by an epithelium expressing hair-related keratins (Dhouailly et al., 1989). The information on the tongue papillae development presented in this review was obtained from previous experiments in sheep, rodents and salamanders as well as from studies in human development (Paulson et al., 1985; Barlow and Northcutt, 1995; Iwasaki et al., 1996; Witt and Reutter, 1997). The initial study using a vertebrate tongue was aimed at examining the three-dimensional structure of the embryonic and fetal stages of the tongue and its appendages, the tongue papillae, using light microscopy and scanning electron microscopy (Paulson et al., 1985; Iwasaki et al., 1996). It was reported that during the development of the tongue papillae many factors might be involved in morphogenesis, including the ECM (extracellular ma-

Abbreviations used in this paper: CAM, cell adhesion molecule; ECM, extracellular matrix.

\footnotetext{
*Address correspondence to: Dr. Han-Sung Jung. Division of Anatomy and Developmental Biology, Dept. of Oral Biology, College of Dentistry, Yonsei University, 134 Shinchon-Dong, Seodaemoon-Gu, Seoul, 120-752, Korea. Fax: +82-2-312-8012. e-mail: hsjung@yumc.yonsei.ac.kr
} 
trix) molecules such as laminin, CAM (Cell adhesion molecule) and cytokeratins (Sawaf et al., 1990; Nolte and Martini, 1992; Mistretta and Haus, 1996). Recently, the circumvallate and fungiform papillae were examined using a variety of experimental approaches including protein expression, signalling network and tissue interactions (Jitpukdeebodintra et al., 2002; Hall et al., 2003; Kim et al, 2003). In particular, the development of fungiform papillae, which are one of the gustatory papillae, was examined as an epithelial appendage (Chuong et al, 2000). In this study, the formation of the fungiform papillae is discussed as a model system for pattern formation because they have a specific patterned array on the dorsal surface of the tongue (Oakley, 1998; Barlow, 1999; Mistretta et al., 2003).

\section{Outline of tongue and gustatory papillae development in vertebrates}

During the initial stages of organogenesis, the induction and formation of the epithelial appendages, including feathers, hairs, mammary glands and teeth require specific and complex epithelial-mesenchymal interactions throughout their development (Sengel, 1976; Pispa and Thesleff, 2003; Veltmaat et al., 2003). Although the vertebrate epithelial appendages have specific structures and diverse functions, most epithelial appendages have similar developmental stages, including induction, morphogenesis and differentiation (Dhouailly, 1977; Chuong, 1998).

In feathers or in scale forming regions of the skin of chick embryos, the epidermal placode is the first morphological event
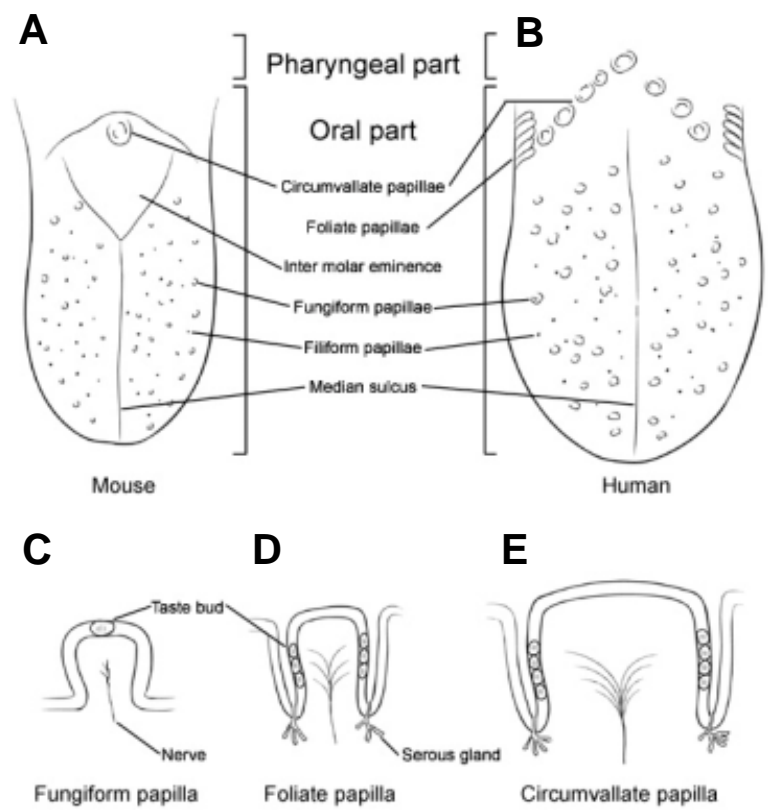

Fig. 1. Schematic diagrams of the mouse and human tongue. (A) The mouse tongue and the general boundaries of the oral part, the pharyngeal part and the intermolar eminence of the tongue. Mice have a single circumvallate papilla in the middle of the posterior part of the tongue. (B) The human tongue illustrating the location of the fungiform papillae, foliate papillae and circumvallate papillae. (C) The fungiform papilla. The taste bud is localized in the apex of the papilla. (D) The foliate papilla. (E) The circumvallate papilla. for the formation of an epithelial appendage. It provides a signal to the dermis, which results in the formation of dermal condensations immediately beneath the placode (Viallet et al, 1998). The formation of the epidermal placodes is determined by the mesodermal signals, which was revealed by the heterospecific tissue recombinations of the ectodermal and mesodermal components of the embryonic skin (Dhouailly, 1973). Furthermore, the mesenchyme determines the number, size, location as well as the structural identity of the appendages, while the epithelium determines the zoological class type (feathers or hair) and the competence state (Dhouailly, 1977; Chuong et al, 1996; Jiang et al., 1999). Therefore, the molecular mechanisms are conserved during the evolution of the skin as well as another developmental model system such as the limbs. Moreover, similar molecules appear to be essential for its proper development (Tickle, 2003). The development of the gustatory papillae has similar pattern of formation with the other epithelial appendages. The fungiform papillae also form a repeating unit with a highly regular spatial pattern through the epithelial-mesenchymal interaction over the dorsal surface of the tongue during embryonic development. A recent study reported that a reciprocal interaction between the tongue epithelium and the underlying mesenchyme is of fundamental importance in determining the individual position of the fungiform papilla as well as its stage-dependent competency (Kim et al., 2003). In mice, the fungiform papillae begin to develop as an epithelial thickening at E13. As the tongue develops, the mesenchymal cells become more rounded and closely apposed to form a condensate. The connective tissue is overlaid by an epithelium, which is two cells thick. The periderm cells, which form a superficial thin sheet of cells, are formed over the epithelium in early stage. During fungiform papillae development, the epithelial cells elongate in the primordium and thicken (Fig. 2A) (Jung et al., 1999; Kim et al., 2003). The tongue epithelium and mesenchyme undergo subsequent morphogenesis (Fig. 2 B-D) with a stereotyped pattern until E17 (Fig. 2 E,F). Previous studies have suggested that the morphogenesis of the gustatory papillae requires a series of invaginations and evaginations, which are coordinated interactions among the lingual epithelium and mesenchyme as well as in the nerve innervation of the tongue (Barlow et al., 1996; Hall et al., 1999). The development of the fungiform papillae can be divided into two phases. The first phase is the morphogenesis phase, in which the papillae structures are produced. The second phase is the innervation phase, in which the taste buds develop. In the innervation phase, the fungiform papillae display a different developmental procedure for complete development than the other epithelial appendages. In the case of the fungiform papilla, the nerve innervation is required to develop into sensory organs with taste buds, as shown during the development of mice (Oakley, 1998; Hall et al., 1999; Barlow 1999). Therefore, the innervation phase represents a maturation phase or functional phase (Kim et al., 2003). Jitpukdeebodintra et al., after examining the cell adhesion molecules, reported that the formation of the circumvallate papilla initially depends on cell migration, which likely to play a major role during circumvallate placode formation (Jitpukdeebodintra et al., 2002). During the initial tongue innervation, the nerves follow a spatiotemporary restricted pathway, suggesting that neural guidance is governed by signaling molecules from environmental factors such as BDNF (Brain derived neurotrophic factor), NT4 (Neurotrophin-4), E- 
cadherin and laminin (Barlow et al., 1996; Witt an Reuter, 1997; Mbiene and Mistretta, 1997). Indeed, as a special sensory organ, the gustatory papillae are closely associated with nerve innervation, which has been examined in detail studies on taste buds (Dulac, 2000).

\section{Pattern formation and molecular studies in gustatory papillae development}

One of the simplest and frequently observed embryonic patterns is the maintenance of a minimum distance between the repetitive neighboring elements, namely the spacing patterns (Wolpert, 1998). The spacing patterns are of fundamental importance in the various repeated structures, which develop at regular intervals. The development of the mouse gustatory papillae provides a good model for examining pattern formation (Mbiene et al., 1997). In mice, the fungiform papillae have been observed to form a specific patterned array (Jung et al., 1999).

In chicks, feather formation in the dorsal skin results in the formation of a hexagonal patterned array of feather buds (Sengel, 1976). Prior to the morphological changes, a highly ordered pattern of expression of signaling molecules such as Shh, Bmps, $F g$ s, etc, is found. These molecules participate in skin morphogenesis (Bennett et al., 1995; Jung et al., 1999; Hall et al., 1999; Chuong etal., 2000; Huang etal., 2001; Hall et al., 2003; Mistretta et al., 2003).

What roles do the signaling molecules play during fungiform papillae development? Recent studies of the fungiform papillae in developing mouse embryos have shown that the Shh, Ptc, Gli-1, $B m p-2, B m p-4$ and $F g f-8$ transcripts are expressed in the fungiform papillae forming area prior to the morphological changes, indicating that they are implicated in papillae morphogenesis (Fig. 3) (Jung et al, 1999; Hall et al., 1999). In particular, Shhplays critical roles in patterning the lingual epithelium (Hall et al., 2003; Mistretta et al., 2003). Shh is involved in several developmental processes and can act as a polarizing signal during limb bud development (Khokha et al., 2003). It can also act as an inductive signal in the neural tube, inducing the development of the lingual epithelium during the life of the papillary cells (Ishibashi and McMahon, 2002). Shh might be active in the epithelial-mesenchymal interaction or during the establishment of the papillary spacing pattern. Interestingly, it has been shown in mice that there is a relationship between innervation and the level of expression of Shh during fungiform papillae development (Hall et al., 1999). In addition, the $\mathrm{SHH}$ protein is involved in the morphogenesis and pattern formation of the tongue papillae as a morphogen, which directs the cells toward a nonpapillary fate and as a mitogen, which causes the expansion of the interplacodal epithelium (Hall et al., 2003). Disruption of Shh signaling during rat tongue development revealed that the development and patterning of the fungiform papillae depends on this signaling pathway (Mistretta et al., 2003). In mice, at E11, Shh is expressed in the entire tongue epithelium. At E12 to 13, Shh expression is restricted to individual spots. Shh continues to be expressed in the developing fungiform papillae in discrete spot patterns at E14 and E15 (Fig. 2 E-G). Overall, Shh is required during the initiation, determination and differentiation of fungiform papillae. Similarly, Ptc, Gli-1, Bmp-2 and Bmp-4 are expressed in the epithelium and share a similar expression pattern at regular intervals. In addition, they are located in stereotyped rows at both
A
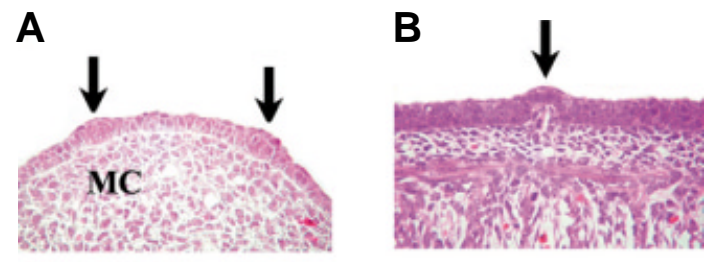

C

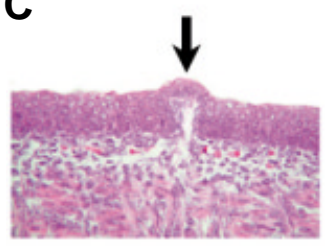

D

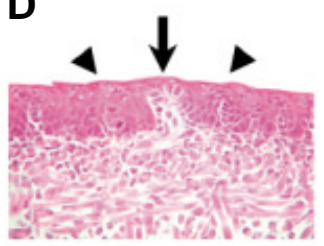

$\mathbf{E}$

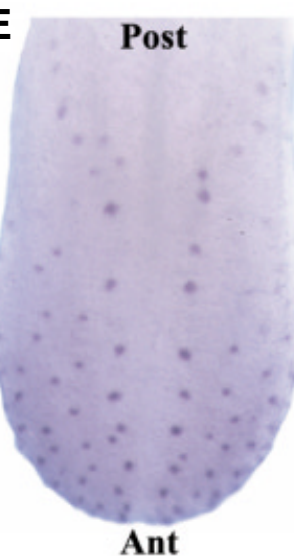

F Dor

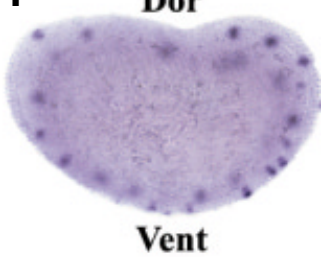

$\mathbf{G}$

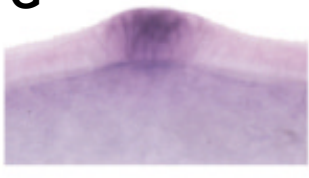

Fig. 2. Morphogenesis and expression of Shh during fungiform papillae development. (A-D) Histology of the fungiform papillae during the morphogenesis phase. All the sections were stained with HematoxylinEosin. (A) At E13, epithelial thickenings (arrows) were observed. The mesenchymal cells were condensed under the epithelial thickening IMC, mesenchymal condensation). (B) At E14, the epithelial thickening under the surface region became concave as the condensed mesenchymal cells migrated upward. (C) At E16, the epithelial cells continued to increase in height and filiform papillae began to develop. (D) At E17, the fungiform papillae (arrow) developed between the filiform papillae (arrowhead). (E-G) The expression pattern of Shh in the fungiform papillae at E14 mouse embryo. (E) After whole mount in situ hybridization, Shh expression can be observed in the fungiform papillae with a stereotyped pattern. The dotted line indicates frontal vibratome section in (F) (Ant, anterior; Post, posterior). (F) Frontal vibratome section after the whole mount in situ hybridization (Dor, dorsal; Vent, ventral). (G) High magnification of (F), Shh is expressed in the epithelium, which is a forming papilla.

sides of the median sulcus prior to the morphological changes. Shh continues to be expressed in the developing fungiform papillae in discrete spot patterns at E14 and E15 (Fig. 2 E-G). Although the temporal sequence of papilla development has been established in several species, the mechanisms involved in papilla differentiation, patterning, growth and morphogenesis are not elucidated (Bao etal., 1995; Chou etal., 2001; Krimm etal., 2001; Mbiene and Roberts, 2003; Mistretta et al., 2003). It should be noted that the signalling molecules, growth factors, transcription factors, cell surface receptors and extracellular matrix proteins, which have been implicated in the morphogenetic regulation of many other epithelial appendages, are also associated with the initiation of the tongue papillae formation. 


\section{Specificity for studying a model system of develop- mental biology and future directions}

The development of the epithelial appendages has been examined intensively over the last 30 years, mainly using a number of model systems such as feathers, hair, teeth, as well as the mammary and salivary glands (Sengel, 1976; Dhouailly, 1993; Chuong et al., 1998; Hogan and Yingling, 1998; Kuure et al., 2000; Millar, 2002; Thesleff and Mikkola, 2002; Pispa and Thesleff, 2003; Veltmaat $e t$ al., 2003). The fungiform papillae might be another new model system for examining epithelial appendage development. All these model systems share similar developmental processes in the early embryonic stages. In particular, fungiform papillae development shows some similarities with the development of other epithelial appendages. First, prior to the morphological changes, the signaling molecules are expressed in the fungiform papillae forming area with a specific patterned array. Second, the morphogenesis of the fungiform papillae has a specific structure such as epithelial thickening and mesenchymal condensation, which are observed in the morphogenesis of the other epithelial appendages. Third, the stereotyped pattern is present during fungiform papillae development (Mistretta, 1991). Fourth, the fungiform papillae develop through reciprocal interactions between epithelial and mesenchymal tissue. It would be interesting to know how, in the case of the gustatory papillae, individual position, size and the spacing pattern are determined. Moreover, fungiform papillae have different developmental procedures from the other epithelial appendages. Nerve innervation is required for the development of mouse embryo taste buds (Hall etal., 1999; Mistretta et al., 2003), whereas the fungiform papillae can develop independently of nerve innervation in salamanders (Oakley, 1998; Barlow 1999). This result suggested that nerve innervation is not essential for the initiation of fungiform papillae (Kim et al., 2003), but rather for the complete development of the functional and matured fungiform papillae as sensory organs in mammals. Therefore, an investigation of the primary induction system for the indi-

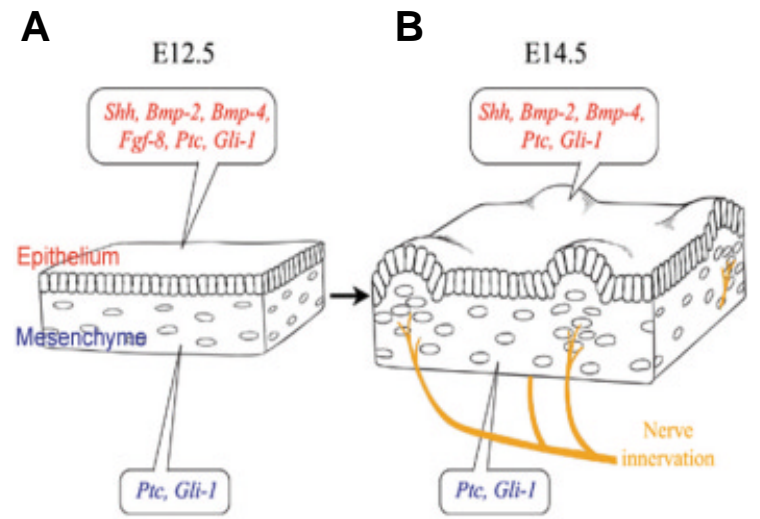

Fig. 3. Schematic diagram showing the signaling molecules which take part in epithelial-mesenchymal interactions at an early stage of morphogenesis of the fungiform papillae. (A) At E12.5, there are no morphological changes. However, expression of the signaling molecules was detected in the epithelium (epithelial signaling: Shh, Bmp-2, Bmp-4, Fgf-8, Ptc, Gli-1) and in the mesenchyme (mesenchymal signaling: Ptc, Gli1). (B) At E14.5, fungiform papillae were structurally developed. Therefore, they are committed to the next developmental phase of nerve innervations to develop the taste buds (epithelial signaling: Shh, Bmp-4, Ptc, Gli-1; mesenchymal signaling: Ptc, Gli-1; Nerve innervation indicated in yellow). vidual papilla should be performed. In addition, the induction of the ectopic papillae as a new study field would be another challenge. A culture system of the mouse tongue has allowed in our laboratory an investigation into the participation of three tissue types, the epithelium, mesenchyme and neural tissue during the initiation, morphogenesis, growth and maintenance of the fungiform papillae (Kim et al., 2003). Concerning evolution, in order to understand the diversity of the fungiform papillae and how it is conserved, a comparative study using other molecular markers and available approaches is suggested. The development of fungiform papillae represents an ideal model system for examining the epithelial-mesenchymal interaction, nerve innervation and spacing pattern formation during embryonic development. Developmental biology perspectives will help throw light on our understanding of the evolution of the fungiform papillae in vertebrates.

\section{Acknowledgements}

The authors wish to acknowledge our lab members for their many helpful suggestions, especially Mr. Kwan-Hyun Youn for the schematic figures. This work was supported by grant No. R01-2003-000-11649-0 from Korea Science \& Engineering Foundation.

\section{References}

BAO, H., BRADLEY, R.M. and MISTRETTA, C.M. (1995). Development of intrinsic electrophysiological properties in neurons from the gustatory region of rat nucleus of solitary tract. Brain Res. Dev. Brain. Res. 86: 143-154.

BARLOW, L.A. and NORTHCUTT, R.G. (1995). Embryonic origin of amphibian taste buds. Dev. Biol. 169: 273-285

BARLOW, L.A., CHIEN, C. and NORTHCUTT R.G. (1996). Embryonic taste buds develop in the absence of innervation. Development. 122: 1103-1111.

BARLOW, L.A. and NORTHCUTT, R.G. (1998). The role of innervation in the development of taste buds: insights from studies of amphibian embryos. Ann. N. Y. Acad. Sci. 855: 58-69.

BARLOW, L.A. (1999). A taste for development. Neuron. 22: 209-212.

BENNETT, J.H., HUNT, P. and THOROGOOD, P. (1995). Bone morphogenetic protein-2 and -4 expression during murine orofacial development. Arch. Oral. Biol. 40(9): 847-854.

CHOU, H.C., CHIEN, C.L. and LU, K.S. (2001). The distribution of PGP9. 5, BDNF and NGF in the vallate papilla of adult and developing mice. Anat. Embryol. (Berl). 204(2): 161-169.

CHUONG, C.M., WIDELITZ, R.B., TING-BERRETH, S. and JIANG, T.X. (1996) Early events during avian skin appendage regeneration: dependence on epithelial-mesenchymal interaction and order of molecular reappearance. J. Invest. Dermatol. 107(4): 639-646.

CHUONG, C.M., JUNG, H.S., NODEN, D. and WIDELITZ, R.B. (1998). Lineage and pluripotentiality of epithelial precursor cells in developing chicken skin. Biochem. Cell Biol. 76(6): 1069-1077.

CHUONG, C.M., PATEL, N., LIN, J., JUNG, H.S. and WIDELITZ, R.B. (2000). Sonic hedgehog signaling pathway in vertebrate epithelial appendage morphogenesis: perspectives in development and evolution. Cell. Mol. Life. Sci. 57(12): 1672-1681.

DHOUAILLY, D. (1973). Dermo-epidermal interactions between birds and mammals: differentiation of cutaneous appendages. J. Embryol. Exp. Morphol. 30(3): 587-603.

DHOUAILLY, D. (1977). Dermo-epidermal interactions during morphogenesis of cutaneous appendages in amniotes. Front Matrix Biol. 4:86 - 121.

DHOUAILLY, D., XU, C., MANABE, M., SCHERMER, A. and SUN, T.T. (1989). Expression of hair-related keratins in a soft epithelium: subpopulations of human and mouse dorsal tongue keratinocytes express keratin markers for hair-, skinand esophageal-types of differentiation. Exp. Cell. Res. 181(1): 141-158.

DHOUAILLY, D. (1993). Genetic expression and morphogenesis of the skin in vertebrates. Ann. Genet. 36(1): 47-55. 
DULAC, C. (2000). The physiology of taste, vintage 2000. Cell. 100(6): 607-610

HALL, J.M., HOOPER, J.E. and FINGER, T.E. (1999). Expression of sonic hedgehog, patched and Gli1 in developing taste papillae of the mouse. J. Comp. Neurol. 406(2): 143-155.

HALL, J.M., BELL, M.L. and FINGER, T.E. (2003). Disruption of sonic hedgehog signaling alters growth and patterning of lingual taste papillae. Dev. Biol.255(2): 263-277.

HOGAN, B.L. and YINGLING, J.M. (1998). Epithelial/mesenchymal interactions and branching morphogenesis of the lung. Curr. Opin. Genet. Dev. 8: 481-486.

HUANG, R., LANG, E.R., OTTO, W.R., CHRIST, B. and PATEL, K. (2001). Molecular and cellular analysis of embryonic avian tongue development. Anat. Embryol. (Berl). 204(3): 179-187.

ISHIBASHI, M. and MCMAHON, A.P. (2002). A sonic hedgehog-dependent signaling relay regulates growth of diencephalic and mesencephalic primordia in the early mouse embryo. Development. 129(20): 4807-4819.

IWASAKI, S., ASAMI, T. and WANICHANON, C. (1996). Ultrastructural study of the dorsal lingual epithelium of the soft-shell turtle, Trionyx cartilagineus (Chelonia, Trionychidae). Anat. Rec. 246(3): 305-316.

IWASAKI, S., YOSHIZAWA, H. and KAWAHARA, I. (1999). Ultrastructural study of the relationship between the morphogenesis of filiform papillae and the keratinisation of the lingual epithelium in the rat. J. Anat. 195 (Pt 1), 27-38.

JITPUKDEEBODINTRA, S., CHAI, Y. and SNEAD, M.L. (2002). Developmental patterning of the circumvallate papilla. Int. J. Dev. Biol. 46(5): 755-763.

JIANG, T.X., JUNG, H.S., WIDELITZ, R.B. and CHUONG, C.M. (1999). Selforganization of periodic patterns by dissociated feather mesenchymal cells and the regulation of size, number and spacing of primordia. Development.126(22): 4997-5009.

JUNG, H.S., OROPEZA, V. and THESLEFF, I. (1999). Shh, Bmp-2, Bmp-4 and Fgf8 are associated with initiation and patterning of mouse tongue papillae. Mech. Dev. 81(1-2): 179-182.

KHOKHA, M.K., HSU, D., BRUNET, L.J., DIONNE, M.S. and HARLAND, R.M. (2003). Gremlin is the BMP antagonist required for maintenance of Shh and Fgf signals during limb patterning. Nat. Genet. 34(3): 303-307.

KIM, J.Y., MOCHIZUKI, T., AKITA, K. and JUNG, H.S. (2003). Morphological Evidence of the Importance of Epithelial Tissue during Mouse Tongue Development. Exp. Cell Res. 290: 217-226.

KRIMM, R.F., MILLER, K.K., KITZMAN, P.H., DAVIS, B.M. and ALBERS, K.M. (2001). Epithelial overexpression of BDNF or NT4 disrupts targeting of taste neurons that innervate the anterior tongue. Dev. Biol. 232(2): 508-521.

KUURE, S., VUOLTEENAHO, R. and VAINIO, S. (2000). Kidney morphogenesis: cellular and molecular regulation. Mech. Dev. 92: 31-45.

MBIENE, J.P. and MISTRETTA, C.M. (1997). Initial innervation of embryonic rat tongue and developing taste papillae: nerves follow distinctive and spatially restricted pathways. Acta. Anat. (Basel). 160(3): 139-158.

MBIENE, J.P., MACCALLUM, D.K. and MISTRETTA, C.M. (1997). Organ cultures of embryonic rat tongue support tongue and gustatory papilla morphogenesis in vitro without intact sensory ganglia. J. Comp. Neurol. 377(3): 324-340.
MBIENE, J.P. and ROBERTS, J.D. (2003). Distribution of keratin 8-containing cell clusters in mouse embryonic tongue: evidence for a prepattern for taste bud development. J. Comp. Neurol. 457(2): 111-122.

MILLAR, S.E. (2002). Molecular mechanisms regulating hair follicle development. J. Invest. Dermatol. 118: 216-225.

MISTRETTA, C.M. (1991). In Developmental Neurobiology of the Taste System. Taste and Smell in Health and Disease. (Ed. Getchell, T.V). Raven Press, New York, pp. 35-64.

MISTRETTA, C.M. and HAUS, L.F. (1996). Temporal and spatial patterns of tenascin and laminin immunoreactivity suggest roles for extracellular matrix in development of gustatory papillae and taste buds. J. Comp. Neurol. 364(3): 535555.

MISTRETTA, C.M., LIU, H.X., GAFFIELD, W. and MACCALLUM, D.K. (2003). Cyclopamine and jervine in embryonic rat tongue cultures demonstrate a role for Shh signaling in taste papilla development and patterning: fungiform papillae double in number and form in novel locations in dorsal lingual epithelium. Dev. Biol. 254(1): 1-18.

NOLTE, C. and MARTINI, R. (1992). Immunocytochemical localization of the L1 and $\mathrm{N}-\mathrm{CAM}$ cell adhesion molecules and their shared carbohydrate epitope L2/HNK1 in the developing and differentiated gustatory papillae of the mouse tongue. $\mathrm{J}$. Neurocytol. 21(1): 19-33.

OAKLEY, B. (1998). Taste neurons have multiple inductive roles in mammalian gustatory development. Ann. N. Y. Acad. Sci. 855: 50-57.

PAULSON, R.B., HAYES, T.G. and SUCHESTON, M.E. (1985). Scanning electron microscope study of tongue development in the CD-1 mouse fetus. J. Cranio. Gene. Dev. Biol. 5(1): 59-73.

PISPA, J. and THESLEFF, I. (2003). Mechanisms of ectodermal organogenesis. Dev. Biol. 262:195-205.

SAWAF, M.H., OUHAYOUN, J.P., SHABANA, A.H. and FOREST, N. (1990). Cytokeratin expression in human tongue epithelium. Am. J. Anat.189(2): 155-166.

SENGEL, P. (1976). In Morphogenesis of skin. (Eds. Abercrombie, M., Newth, D.R and Torrey, J.G.). Cambridge University Press, Cambridge.

THESLEFF, I. and MIKKOLA, M.L. (2002). The role of growth factors in tooth development. Int. Rev. Cytol. 217: 93-135.

TICKLE, C. (2003). Patterning system- from one end of the limb to the other. Dev. Cell. 4(4): 449-458.

VIALLET, J.P., PRIN, F., OLIVERA-MARTINEZ, I., HIRSINGER, E., POURQUIE, O and DHOUAILLY, D. (1998). Chick Delta-1 gene expression and the formation of the feather primordia. Mech. Dev. 72(1-2): 159-168.

VELTMAAT, J.M., MAILLEUX, A.A., THIERY, J.P. and BELLUSCI, S. (2003). Mouse embryonic mammogenesis as a model for the molecular regulation of pattern formation. Differentiation. 71: 1-17.

WITT, M. and REUTTER, K. (1997). Scanning electron microscopical studies of developing gustatory papillae in humans. Chem. Senses. 22(6): 601-612.

WOLPERT, L. (1998). Pattern formation in epithelial development: the vertebrate limb and feather bud spacing. Philos. Trans. R. Soc. Lond. B. Biol. Sci. 353(1370): 871-875 